\title{
Spontaneous fission of rutherfordium isotopes - total kinetic energies
}

\author{
Pavol Mosat ${ }^{1,2, *}$, Fritz Peter Heßberger ${ }^{3,4}$, Dieter Ackermann ${ }^{3,5}$, Boris Andel ${ }^{1,6}$, Stanislav Antalic ${ }^{1}$, Michael Block ${ }^{3,4,7}$, \\ Sigurd Hofmann ${ }^{3,8}$, Zdenka Kalaninova ${ }^{1,9}$, Birgit Kindler $^{3}$, Mustapha Laatiaoui ${ }^{3,4}$, Bettina Lommel $^{3}$, Andrew Mistry ${ }^{3,4}$, \\ Katsuhisa Nishio ${ }^{10}$, Julien Piot $^{5}$, Barbara Sulignano ${ }^{3,11}$, and Marija Vostinar $^{5}$ \\ ${ }^{1}$ Department of Nuclear Physics and Biophysics, Comenius University in Bratislava, 84248 Bratislava, Slovakia \\ ${ }^{2}$ Flerov Laboratory of Nuclear Reactions, JINR, 141980 Dubna, Russia \\ ${ }^{3}$ GSI Helmholtzzentrum für Schwerionenforschung, GmbH, 64291 Darmstadt, Germany \\ ${ }^{4}$ Helmholtz - Institut Mainz, 55099 Mainz, Germany \\ ${ }^{5}$ GANIL, 14076 Caen CEDEX 5, France \\ ${ }^{6} \mathrm{KU}$ Leuven, Instituut voor Kern- en Stralingsfysica, B-3001 Leuven, Belgium \\ ${ }^{7}$ Johannes Gutenberg-Universität Mainz, 55128 Mainz, Germany \\ ${ }^{8}$ Institut für Physik, Goethe-Universität Frankfurt, 60054 Frankfurt, Germany \\ ${ }^{9}$ Laboratory of Nuclear Problems, JINR, 141980 Dubna, Russia \\ ${ }^{10}$ JAEA, Tokai, Ibaraki 319-1195 Japan \\ ${ }^{11}$ CEA Saclay, IRFU, SPhN, 91191 Gif-sur-Yvette, France
}

\begin{abstract}
The isotopes ${ }^{255,256,258} \mathrm{Rf}$ were produced in the fusion-evaporation reactions ${ }^{50} \mathrm{Ti}+{ }^{207,208} \mathrm{~Pb}$ and ${ }^{50} \mathrm{Ti}+{ }^{209} \mathrm{Bi}$ at GSI Darmstadt, using the velocity filter SHIP. Total kinetic energies of fragments from spontaneous fission for these isotopes were evaluated with a correction to pulse-height defect.
\end{abstract}

\section{Introduction}

Spontaneous fission (SF) is a decay mode with large impact on the stability of nuclei in the transfermium region. These isotopes are stabilized against SF mainly by microscopic shell effects [1]. Systematic studies of SF properties allow us to understand these effects and determine the production possibilities for the heaviest nuclei. Up to now, only a few results (often based on limited statistics of SF events) with measured total kinetic energy (TKE) were obtained for rutherfordium $(Z=104)$ isotopes [2-5].

\section{Experiment}

The experiment was performed at GSI Darmstadt in Germany. The heavy-ion ${ }^{50} \mathrm{Ti}$ beam with typical energies from 225 to $243 \mathrm{MeV}$ was delivered by the UNILAC accelerator. The fusion-evaporation reactions ${ }^{50} \mathrm{Ti}+{ }^{207,208} \mathrm{~Pb}$ were used for production of the studied isotopes ${ }^{255,256} \mathrm{Rf}$ via $\mathrm{x} n$ evaporation channels from the compound nuclei ${ }^{257} \mathrm{Rf}$ and ${ }^{258} \mathrm{Rf}$. The reaction ${ }^{50} \mathrm{Ti}+{ }^{209} \mathrm{Bi}$ was used for indirect production of ${ }^{258} \mathrm{Rf}$ via EC decay of ${ }^{258} \mathrm{Db}$.

Evaporation residues (ER) were separated from the beam and other undesired background using the velocity filter SHIP [6] and delivered to a detection setup. After passing through the time-of-flight system they were implanted $(6.5-6.8) \mu \mathrm{m}$ deep into the 16-strip positionsensitive STOP detector. The same-type detectors arranged in the "BOX" geometry were placed in front of the

*e-mail: pavol.mosat@fmph.uniba.sk
STOP detector to detect escaping particles. A clover detector with four Ge crystals was placed behind the STOP detector for $\gamma$ - and X-ray detection.

\section{Results}

In order to detect SF events from specific isotope, we used a time and position correlation search technique. In the case of ${ }^{255} \mathrm{Rf},{ }^{256} \mathrm{Rf}$ we searched for correlations between an ER implantation signal and a high-energy signal corresponding to SF. For ${ }^{258} \mathrm{Rf}$ instead of the ER implantation signal we used the low-energy signal corresponding to electrons originating from internal conversion process from de-excitation of levels populated after the EC decay ${ }^{258} \mathrm{Db} \stackrel{E C}{\longrightarrow}{ }^{258} \mathrm{Rf}$. The time conditions were set to $\approx 5 \times$ half-life between signals for each isotope $(8500 \mathrm{~ms}$ for ${ }^{255} \mathrm{Rf}, 35 \mathrm{~ms}$ for ${ }^{256} \mathrm{Rf}$ and $60 \mathrm{~ms}$ for ${ }^{258} \mathrm{Rf}$ ) and position window of $1 \mathrm{~mm}$ in the detector for ${ }^{255} \mathrm{Rf}$ and ${ }^{256} \mathrm{Rf}$. As position condition for ${ }^{258} \mathrm{Rf}$, we required only the same strip number since for many low-energy signals from electrons the position information was not registered. We collected several hundreds of SF events for ${ }^{255} \mathrm{Rf},{ }^{256} \mathrm{Rf}$ and ${ }^{258} \mathrm{Rf}$. The TKE distributions of fission fragments for each isotope are shown in Fig. 1. The histograms contain all registered SF events - the cases when both fragments stayed in the STOP detector ( $\approx 60 \%$ ) as well as events when one fragment escaped the STOP detector and was either detected in the BOX detector $(\approx 30 \%)$ or escaped the detection setup completely $(\approx 10 \%)$. 


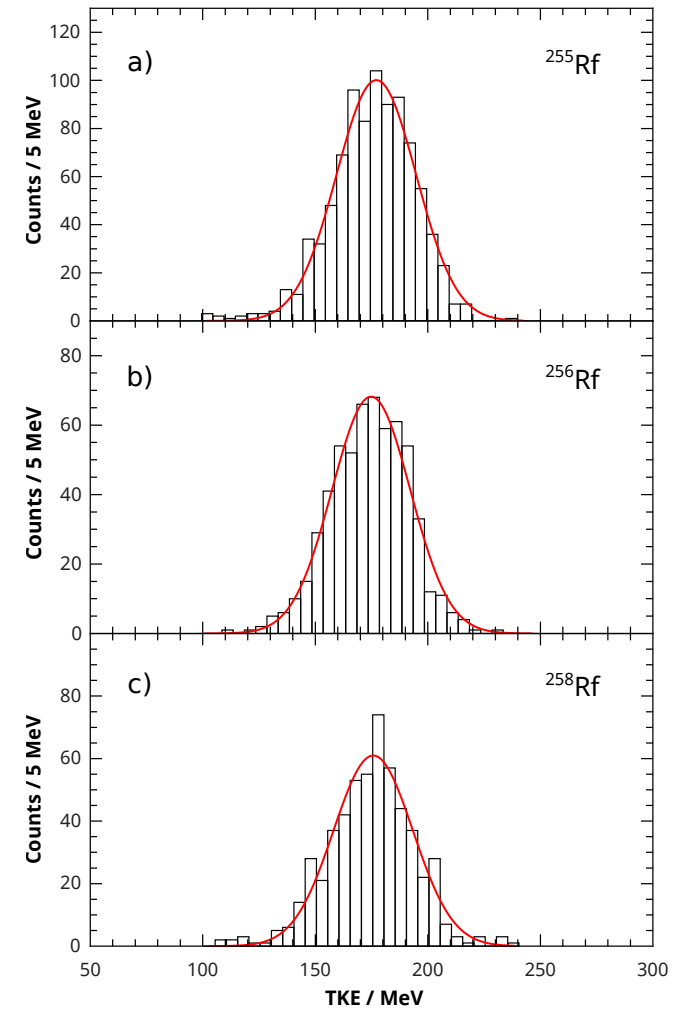

Figure 1. Total kinetic energies for ${ }^{255} \mathrm{Rf},{ }^{256} \mathrm{Rf}$ and ${ }^{258} \mathrm{Rf}$ from all observed SF events for each isotope. Due to the pulse-height defect, the positions of gaussians are shifted to lower values.

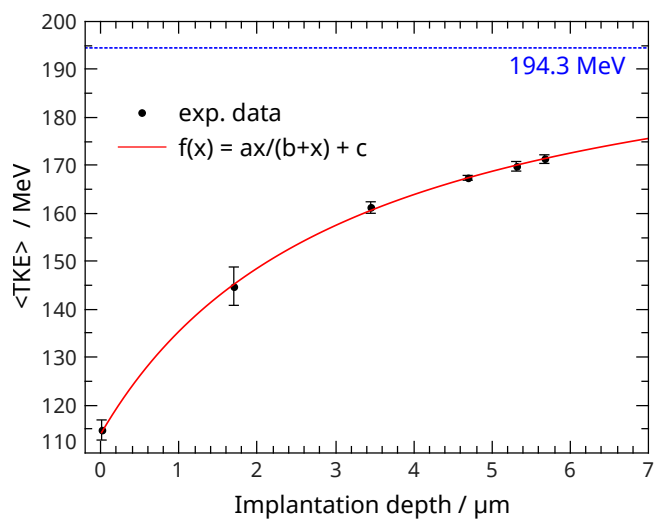

Figure 2. $\overline{T K E}$ from the SF of ${ }^{252} \mathrm{No}$ vs. implantation depth of ER in the detector. Blue dashed line: $\overline{T K E}=194.3 \mathrm{MeV}$ of ${ }^{252}$ No from [3]. Red solid line: saturation-growth fit.

The crucial task for the evaluation of $\overline{T K E}$ using $\mathrm{Si}$ detectors is the correction of deficit in measured energies. There are two main effects influencing the TKE measurements, discussed in previous studies [7-9] performed at SHIP. First, the energy calibration based on $\alpha$-decay energies is not valid for fission fragments due to the pulseheight defect (see e.g. [10]), resulting in a significant energy deficit. The second effect is a dependence of the detected TKE on the implantation depth of ER into the detector. In order to find the energy correction, we performed TKE measurements for ${ }^{252}$ No with known $\overline{T K E}=$
194.3 MeV [3] at six different implantation depths [8]. Our observations (shown in Fig. 2) proved the strong nonlinear saturation-like dependence of detected TKE on implantation depth (see [11] for more details). The correction to the energy deficit at given implantation depth was determined as a difference of the reference $\overline{T K E}$ of $194.3 \mathrm{MeV}$ and the value $\overline{T K E}_{f i t}$ from saturation-growth model fit of the six points (blue dashed and red solid lines in Fig. 2) as $\Delta E=\left(194.3-\overline{T K E}_{f i t}\right) \mathrm{MeV}$.

Table 1. In the table columns, from left to right, the isotope, $\overline{T K E}$ evaluated in this work, reference value of $\overline{T K E}$ and corresponding references are stated.

\begin{tabular}{llll}
\hline Isotope & $\begin{array}{l}\overline{T K E}_{\text {exp }} \\
{[\mathrm{MeV}]}\end{array}$ & $\begin{array}{l}\overline{T K E}_{\text {ref }} \\
{[\mathrm{MeV}]}\end{array}$ & Ref. \\
\hline${ }^{255} \mathrm{Rf}$ & $199.5 \pm 2.7$ & $199 \pm 3$ & {$[4]$} \\
${ }^{256} \mathrm{Rf}$ & $198.7 \pm 2.8$ & $198.9 \pm 4.4$ & {$[2]$} \\
${ }^{258} \mathrm{Rf}$ & $198.2 \pm 3.0$ & $197.6 \pm 1.1$ & {$[2]$} \\
\hline
\end{tabular}

Considering the facts, that ${ }^{252}$ No is close in $Z, A$ and $\overline{T K E}$ to studied $\mathrm{Rf}$ isotopes, we applied this correction to $\overline{T K E}$ of ${ }^{255} \mathrm{Rf},{ }^{256} \mathrm{Rf}$ and ${ }^{258} \mathrm{Rf}$. Corrected $\overline{T K E}$ values for each isotope are summarized in Table 1 and compared to values from previous studies. The results are in a good agreement, which supports the validity of our correction.

\section{Acknowledgments}

We thank the UNILAC staff as well as the ion source crew for delivering beams of high and stable intensity. We are also grateful to J. Steiner, W. Hartmann, and A. Hubner for production of the large area targets. We want to express our gratitude to H.G. Burkhard and J. Maurer for skillful maintenance of the mechanical and electrical components of SHIP. Four of us, BA, SA, PM, ZK, were supported by the Slovak Research and Development Agency (Contracts No. APVV-18-0268 and No. APVV-14-0524) and by the Slovak grant agency VEGA (Contract No. 1/0532/17).

\section{References}

[1] S.G. Nilsson et al., Nucl. Phys. A 131, 1 (1969)

[2] J.F. Wild et al., J. Alloy. Comp. 213, 86 (1994)

[3] E.K. Hulet, Phys. At. Nucl. 57, 1099 (1994)

[4] S. Antalic et al., Eur. Phys. J. A 51, 41 (2015)

[5] A.I. Svirikhin et al., Phys. Part. Nucl. Lett. 13, 480 (2016)

[6] G. Münzenberg et al., Nucl. Instrum. Meth. 161, 65 (1979)

[7] F.P. Heßberger et al., Z. Phys. A 322, 557 (1985)

[8] K. Nishio et al., AIP Conf. Proc. 891, 71 (2007)

[9] S. Hofmann et al., Eur. Phys. J. A 32, 251 (2007)

[10] B. Wilkins et al., Nucl. Instrum. Meth. 92, 381 (1971)

[11] P. Mosat et al., Acta Phys. Pol. B 49, 605 (2018) 